\title{
Ultra-compact (X-ray) binaries
}

\author{
G. Nelemans \\ Department of Astrophysics, IMAPP, Radboud University Nijmegen, NL \\ P.G. Jonker \\ SRON National Institute for Space Research, Utrecht, NL \\ Harvard-Smithsonian Center for Astrophysics, Cambridge, USA
}

\begin{abstract}
A short review of ultra-compact binaries, focused on ultra-compact X-ray binaries, is followed by a discussion of recent results of our VLT campaign to obtain optical spectra of (candidate) ultra-compact X-ray binaries. We find evidence for carbon/oxygen as well as helium/nitrogen discs and no evidence for (traces) of hydrogen. This suggests that the donors in the observed systems are white dwarfs. However, we also find large differences between the two $\mathrm{C} / \mathrm{O}$ discs of which we have good spectra, which highlights the need for a better understanding of the optical spectra.
\end{abstract}

\section{Introduction: ultra-compact binaries}

Ultra-compact binaries are double stars with periods less than about one hour. In such short-period binaries the components must be so close together that ordinary, hydrogen rich, stars do not fit in (e.g. Nelson et al., 1986). Both stars in ultra-compact binaries thus must be compact stars: the cores of evolved giants, white dwarfs, neutron stars or black holes.

Of special interest are ultra-compact binaries in which mass is transferred from one component to the other. Observationally two types are known: ones with an white dwarf accretor (called AM CVn systems, after the first detected system) and ones with a neutron star accretor, called ultra-compact X-ray binaries (UCXBs). There are currently 16 AM CVn stars known, plus two candidate systems. For more information we refer to a recent review (Nelemans, 2005). Four new systems were reported in the last year, found in the SDSS spectroscopic database (Anderson et al., 2005). The number of known systems is increasing rapidly: 9 of the known systems and one of the two candidates were discovered in the last five years. The two candidate systems V407 Vul and RX J0806.3+1527, are both found as X-ray sources, showing a 100 per cent modulation on 
9.5 and 5.4 mins respectively. One interpretation is that these periods are orbital periods and thus they could be part of the AM CVn family. However, their characteristics, in particular the systematic shortening of their periods (Strohmaver, 2004; Hakala et al., 2003; Ramsav et al., 2005; Strohmaver, 2005), are difficult to reconcile with them being AM CVn stars, but see Marsh \& Nelemans (2005). Alternative models that have been proposed are a face-on longerperiod binary in which we only see the spin period of an accreting white dwarf (Norton et al., 2004) and a pair of detached white dwarfs generating X-ray emission through unipolar induction (Wu et al., 2002). The latter has recently attracted new attention, with claims that the original model cannot explain the observations (Marsh \& Nelemans, 2005) as well as suggestions that in a somewhat different form the model can at least explain RX J0806.3+1527 (Dall'Osso et al., 2005).

(Ultra-compact) X-ray binaries are rare objects. The known UCXBs are much further away than the AM CVns stars which hinders optical studies. Therefore it is only with the advent of $8 \mathrm{~m}$ class telescopes that high-quality optical spectra have become available, and consequently much of our current knowledge is based on X-ray observations and photometry at different wavelengths. There are 12 systems with known or suggested orbital periods. Four more systems are identified based on similarities with known systems in either their X-ray spectra (Juett et al., 2001) or as a result of their optical faintness in outburst, as expected for the small accretion discs in these systems van Paradijs \& McClintock, 1994). Six systems are found in a globular cluster (see Kuulkers et al., 2003; Verbunt \& Lewin, 2005, for reviews of globular cluster X-ray sources).

\subsection{Why study ultra-compact bina- ries}

There are still many open questions in the study of ultra-compact binaries which we would like to answer for the following reasons:

(1) Ultra-compact binaries are strong gravitational-wave sources in the low-frequency regime where the ESA/NASA LISA mission will be sensitive.

(2) They are unique astrophysical laboratories because of the combination of compact objects, short period variability and peculiar chemical composition.

(3) They are important test of binary evolution theory, in particular the common-envelope phase.

\section{A short review on ultra- compact X-ray binaries}

\subsection{Observational properties}

An overview of the observational properties of the known and candidate UCXBs is given in Table 1, 
Table 1

Overview of the UCXB (candidates). Data from van Paradiis \& McClintock (1994), Deutsch et al. (2000), Liu et al. (2001), Kuulkers et al. (2003), Poutanen (2005) unless otherwise indicated.

\begin{tabular}{|c|c|c|c|c|c|c|c|c|}
\hline Name & $\begin{array}{l}\text { Period } \\
(\min )\end{array}$ & $\mathrm{P} / \mathrm{T}$ & $\mathrm{B}$ & $\begin{array}{l}\mathrm{d} \\
(\mathrm{kpc})\end{array}$ & $M_{V} \mathrm{lc}$ & $\log \frac{L_{X}}{L_{\operatorname{Edd}}}$ & remark & Ref. \\
\hline $4 \mathrm{U}$ 1820-30 & 11 & $\mathrm{P}$ & $\mathrm{y}$ & 7.6 & 3.7 & -0.7 & NGC 6624 & \\
\hline $4 \mathrm{U} 1543-624$ & $18(?)$ & $\mathrm{P}$ & $\mathrm{n}$ & $?$ & $?$ & $?$ & Ne-rich(?) & 1 \\
\hline $4 \mathrm{U} 1850-087$ & $21(?)$ & $\mathrm{P}$ & $\mathrm{y}$ & 8.2 & 5.1 & -2.4 & NGC 6712 & 2 \\
\hline M 15 X-2 & 23 & $\mathrm{P}$ & $\mathrm{y}$ & 10.3 & $?$ & -2.2 & M 15 & 3,4 \\
\hline XTE J1807-294 & 40 & $\mathrm{~T}$ & $\mathrm{n}$ & $?$ & $?$ & $?$ & msPSR & \\
\hline $4 \mathrm{U} 1626-67$ & 42 & $\mathrm{P}$ & $\mathrm{n}$ & $<4^{a}$ & $>5.5$ & $<-2.3$ & Ne-rich, slow P & SR \\
\hline XTE J1751-305 & 42 & $\mathrm{~T}$ & $\mathrm{n}$ & $?$ & $?$ & $?$ & msPSR & \\
\hline XTE J0929-314 & 44 & $\mathrm{~T}$ & $\mathrm{n}$ & $?$ & $?$ & $?$ & msPSR & \\
\hline NGC 6652 B & $44 ?$ & $\mathrm{P} ?$ & $\mathrm{y} ?$ & 9.6 & 4.7 & -4.7 & MS colours & 5 \\
\hline $4 \mathrm{U}$ 1916-05 & 50 & $\mathrm{P}$ & $\mathrm{y}$ & 9 & 5 & -2 & Dipper & \\
\hline $4 \mathrm{U} 0614+09$ & $50 ?$ & $\mathrm{P}$ & y & $<3$ & $>5.4$ & $<-2$ & Ne-rich(?) & 6,7 \\
\hline XB 1832-330 & $55 ?^{b}$ & $\mathrm{P}$ & $\mathrm{y} ?$ & 9.6 & 3.7 & -2.3 & NGC $6652(\mathrm{~A})$ & 5 \\
\hline XB 0512-401 & $?$ & $\mathrm{P}$ & $\mathrm{y}$ & 12.1 & $5.6^{c}$ & -2.0 & NGC 1851 & \\
\hline 2S 0918-549 & $?$ & $\mathrm{P}$ & $\mathrm{y}$ & 4.2 & 6.9 & -2.5 & Ne-rich(?) & 6 \\
\hline $4 \mathrm{U} 1822-00$ & $?$ & $\mathrm{P}$ & $\mathrm{n}$ & $<20^{d}$ & $>5.5$ & $<-1$ & Ne-rich(?) & \\
\hline XB $1905+000$ & $?$ & $?$ & $\mathrm{y}$ & 8 & 4.9 & -2.1 & & 1 \\
\hline
\end{tabular}

Notes: $a$ Assuming a distance from the plane $z<1 \mathrm{kpc} ; b$ Or its aliases 2.2 and 4.4 $\mathrm{hr} ; c M_{B} ; d$ Assuming a reasonable maximum distance. For $d=8 \mathrm{kpc}, M_{V}=7.5$ and $\log L_{X} / L_{\mathrm{Edd}}=-1.7$

References: 1 Wang \& Chakrabartv (2004) and refs therein ; 2 Homer et al. (1996); 3 Dieball et al. (2005); 4 White \& Angelini (2001); 5 Heinke et al. (2001); 6 Nelemans et al. (2004) and refs therein; 7 O'Brien et al. (2005)

ordered by (suggested) orbital period (if known). The table lists the name, the orbital period, the type of source (persistent versus transient), the occurrence of type I Xray bursts, the distance, absolute V-band magnitude, X-ray luminosity in terms of the Eddington limit (taken as $2 \times 10^{38} \mathrm{erg} / \mathrm{s}$ ), remarks
$(\operatorname{msPSR}=$ millisecond pulsar, for discussion of the meaning of Ne-rich, see Sect. 2.1.1) and references.

\subsubsection{X-ray properties}

UCXBs have so far been studied mainly in X-rays. We can only summarise the results briefly. There 
are two types of UCXBs: persistent and transient systems. According to Delove \& Bildsten (2003) systems with periods longer than about 30 minutes should be transients if the current mass-transfer rate is similar to its long-time average. The observations show that there are at least 2 and possibly 5 persistent systems with periods longer than 30 min. However, especially in comparing persistent and transient sources there are many biases. Interestingly, the three transients all have periods very close together between 40 and $44 \mathrm{~min}$ and all three of them harbour accretion powered millisecond $\mathrm{X}$-ray pulsars.

\section{(millisecond) pulsations}

An intriguing fact is that three of the six known accreting millisecond pulsars reside in UCXBs, e.g. Markwardt et al. (2002); Remillard et al. (2002), while only 16 UCXBs are known. $4 \mathrm{U} 1626-67$ is a peculiar system because it harbours a young neutron star with a $7 \mathrm{~s}$ spin period.

\section{X-ray spectra}

The X-ray spectra lacked resolution until recent observations obtained with the gratings on board the Chandra satellite. The $\mathrm{X}$-ray spectra are difficult to interpret due to the lack of clear emission features (except in the 7s accreting pulsar 4U 1626-67 which shows strong $\mathrm{O}$ and $\mathrm{Ne}$ emission lines in its X-ray spectrum (Schulz et al., 2001)) and contributions of interstellar absorption. A number of UCXBs have been (first) identified based on their X-ray spectra, when Juett et al. (2001) noted a similarity between the spectrum of $4 \mathrm{U} 1850-087$ and three more LMXBs. The common feature in the X-ray spectra was suggested to be due to an enhancement of neon in these systems, however, this is uncertain. These observations were interpreted as evidence for the donors being ONe, or more likely CO white dwarfs in which Ne has sunk to the core in an earlier evolutionary phase and is now exposed, after accretion has peeled-off the outer layers (Yungelson et al., 2002). Recently it has been noted that if it is really the $\mathrm{O} / \mathrm{Ne}$ ratio that is anomalous, and not the Ne abundance itself, this points towards a He white dwarf donor, in which the $\mathrm{O}$ abundance is strongly reduced due to $\mathrm{CNO}$ processing (in't Zand et al., 2005). Interestingly, one system that shows a similar feature in its X-ray spectrum (4U 1556-605 Farinelli et al., 2003), shows strong hydrogen and helium emission in its optical spectrum, suggesting that it is not an UCXB (Nelemans \& Jonker, in prep).

\section{Type I X-ray bursts}

For a number of UCXBs type I Xray bursts have been found, especially for the ones in globular clusters, but that may be a selection effect. The peculiar chemical composition of the accreting material will influence the burst properties and in principle can give an extra tool to study the chemical composition (e.g. Cumming \& Bildsten, 2001). Recently a number of peculiar bursts have been observed in $2 \mathrm{~S} \quad 0918-549$ and $4 \mathrm{U} \quad 0614+09$ (in't Zand et al., 2005; Kuulkers, 2005) which in't Zand et al. (2005) suggest are due to thick layers of $\mathrm{He}$ that are burned. It is clear that there 
is still uncertainty how we should interpret the different pieces of information, because in some cases the bursts and the optical spectra suggest conflicting compositions as we will discuss below!

\subsubsection{Optical and UV properties}

\begin{abstract}
Absolute magnitudes van Paradiis \& McClintock (1994) investigated the absolute magnitudes of LMXBs, based on the assumption that most of the optical flux is generated by X-ray irradiation of the disc. This means that the two dominant factors determining the absolute magnitude of LMXBs are the X-ray luminosity and the size of the disc. They derived a parameter $\Sigma \propto L_{X}^{1 / 2} P_{\text {orb }}^{2 / 3}$ which should correlate with the absolute magnitude. They indeed found a strong correlation. Because of their small disc size UCXBs are expected to be relatively dim. This has served as an indication for systems to be classified as UCXB candidates. Based on the irradiation model of Arons \& King (1993) we calculated the absolute magnitudes of a number of fiducial LMXBs which we compare to the observed systems (Fig. 1). Indeed most sources seem to be consistent with being UCXBs, although NGC 6652 B (see Sect. 2.1.3) is such a faint X-ray source that the irradiated disc model is probably not applicable for this source and the system more likely is a quiescent LMXB (see Heinke et al., 2001).
\end{abstract}

\section{Photometry}

The faintness of the optical counterparts has inhibited high-quality spectra for a long time, but photometric studies have revealed many of the know orbital periods. Recent developments in photometry are the used of ULTRACAM to study 4U 0614+09, yielding a suggested orbital periods of $50 \mathrm{~min}$ (O’Brien et al., 2005). Recent photometry of $4 \mathrm{U}$ 1543-624 yielded a possible orbital period of $18 \mathrm{~min}$ (Wang \& Chakrabartv, 2004).

\section{Spectra}

We recently started a systematic spectroscopic study of (candidate) UCXBs. The main aim was to confirm/reject candidates and to study the chemical composition of the donors stars in these systems, which hold clues to the formation of UCXBs (see Sect. 2.2). The first results are published in Nelemans et al. (2004) and are summarised in Fig. 2. We identified the features in the spectrum of $4 \mathrm{U} 0614+09$ as relatively low ionisation states of carbon and oxygen. This clearly identifies this system as an UCXB and suggests the donor in this system is a carbonoxygen white dwarf. The similarity of the spectrum of 4U 1543-624 suggests it is a similar system, while for $2 \mathrm{~S}$ 0918-549 the spectrum didn't have a high enough $\mathrm{S} / \mathrm{N}$ ratio to draw firm conclusions, but it is also is consistent with being a similar system (and clearly does not show the characteristic strong hydrogen emission lines of low-mass X-ray binaries). We therefore concluded that all these systems are UCXBs. In Sect. 3 we present new results. 

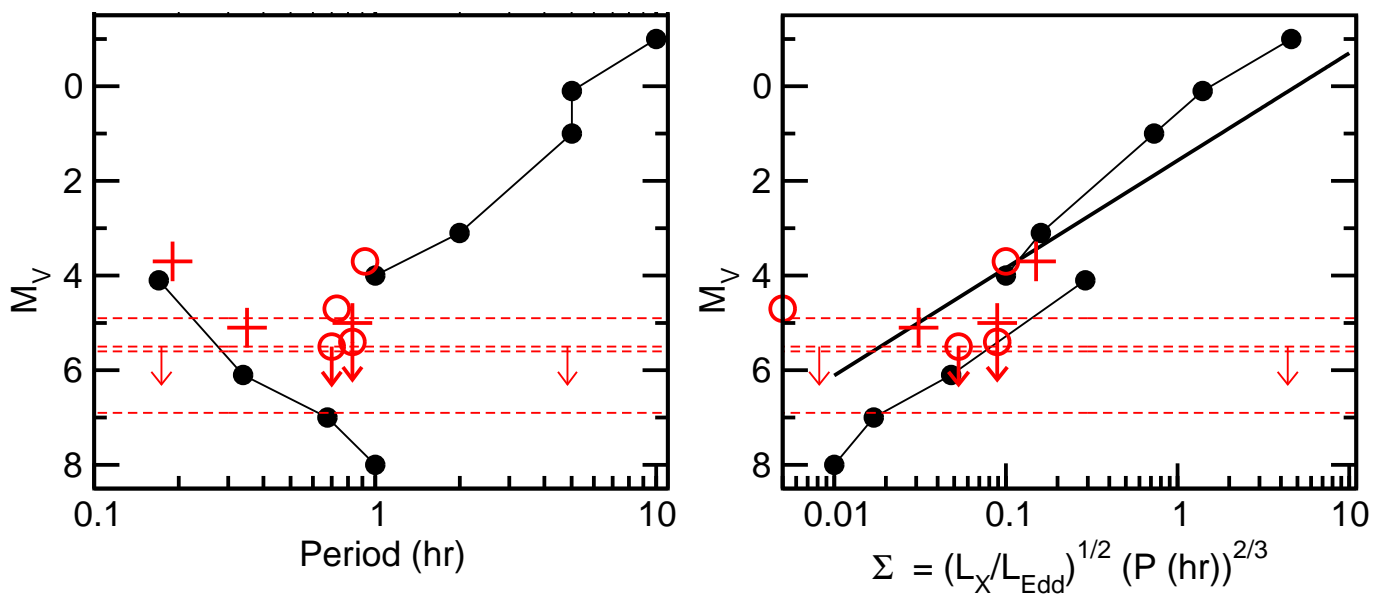

Fig. 1. Absolute magnitudes as function of period (left) and $\Sigma$ (right) for a number of fiducial LMXBs and UCXBs with their mass transfer rate at the long-term average as expected from binary evolution calculations (solid dots). The observed (candidate) UCXBs are indicated with the plusses (period and magnitude reliable), open circles (periods and magnitudes not very reliable) and dashed line (periods unknown). The straight line in the right panel gives the van Paradiis \& McClintock (1994) fit to the observations used by them.

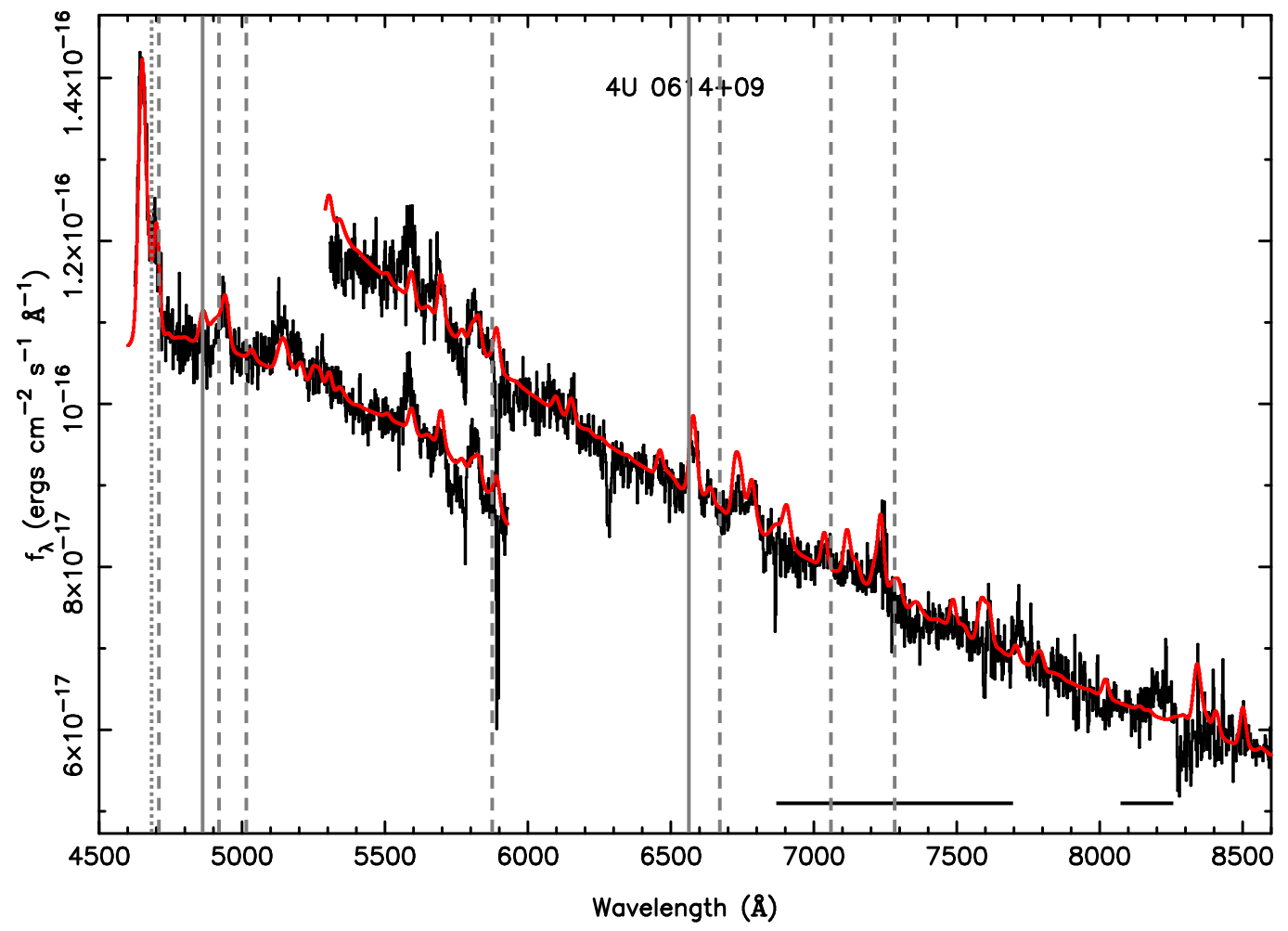

Fig. 2. VLT spectra of $4 \mathrm{U} 0614+09$, showing lines from a carbon-oxygen accretion disc. From Nelemans et al. (2004) 


\subsubsection{Globular cluster sources}

A special mention should be made about UCXBs in globular clusters (GCs). Five of the 12 systems with known orbital periods reside in GCs, an even larger enhancement compared to the disc than for longerperiod X-ray binaries. For reviews of $\mathrm{X}$-ray sources in globular clusters see Verbunt (2005); Verbunt \& Lewin (2005). The shortest period system $4 \mathrm{U}$ 1820-30, which with an orbital period of $11 \mathrm{~min}$ for a long time has been the shortest period binary known and is the most well-known source of this class. The positional accuracy and better sensitivity of Chandra and XMM are clearly crucial for better understanding these systems. For instance in M 15 the bright X-ray source has been resolved in two (White \& Angelini, 2001), the brightest one associated with a faint FUV source that shows a clear modulation on 23 min (Dieball et al., 2005). In NGC 6652 the suggested counterpart of the brightest source turns out to be the counterpart of a (much fainter) X-ray source (source B Heinke et al., 2001). It has a suggested periodicity of $44 \mathrm{~min}$ (Deutsch et al., 2000) but its colours are consistent with a main-sequence star (Heinke et al., 2001). The counterpart of the bright source (source A), has a possible period of $55 \mathrm{~min}$ (but with aliases at 2.2 and $4.4 \mathrm{hr}$ Heinke et al., 2001).

\subsection{Formation and evolution}

For a detailed discussion of the formation of AM CVn systems and UCXBs in the field we refer to Nelemans et al. (2001); Podsiadlowski et al. (2002) and references therein. In short there are three routes, differentiated by the nature of the donor star: (i) a white dwarf donor when a detached binary with a white dwarf and either a neutron star/black hole or white dwarf comes into contact due to angular momentum losses via gravitational-wave radiation; (ii) a (semi-degenerate) helium star donor that evolved from a helium core burning star that filled its Roche lobe to a white dwarf or neutron star/black hole and (iii) the core of a star that filled its Roche lobe to a white dwarf or neutron star/black hole at the end of the main sequence and thus has a helium rich core. In globular clusters the formation of X-ray binaries is probably dominated by dynamical interactions (see Verbunt, 2005).

These different formation scenarios result in principle in different chemical compositions, but there is some overlap. The three formation scenarios will yield the following chemical composition:

(1) White dwarf donors: $\mathrm{He}+\mathrm{CNO}$ processed (i.e. mainly $\mathrm{N}$ ) or $\mathrm{C} / \mathrm{O}$

(2) He star donor: He + He burning products

(3) Evolved secondaries: $\mathrm{He}+\mathrm{CNO}$ processed + some $\mathrm{H}$ 


\subsection{Open questions}

The rapid increase in the number of (candidate) UCXBs in recent years shows that this is an area where new technological developments play an important role. However, with the increase in the sample size a number of new questions have risen, while many of the old questions still remain. A subjective list of the current most important open questions is

- What are the chemical compositions of the donor stars and what are the best measurements to determine this? In particular the interpretation of the optical spectra (see below) and the type I X-ray bursts deserve attention.

- What are the orbital periods of the remaining systems? Only when we have found these periods we can really start to look at the period distribution. The peculiar fact that most systems had an orbital period of about $40 \mathrm{~min}$ is already being diluted by new period determinations at 18 and $23 \mathrm{~min}$. However, still the clustering of the three transients at very similar periods remains.

- What is the determining factor in the difference between transient and persistent sources?

- Why are so many of the accreting millisecond pulsars found in UCXBs? Is this due to the low average mass accretion rate that is expected in the longest-period UCXBs?

\section{VLT spectroscopy of ultra- compact X-ray binaries}

In order to tackle some of the open questions mentioned in the last section, we continued our spectroscopic study of (candidate) UCXBs.

\subsection{Observations}

We used the FORS2 spectrograph on the $8.2 \mathrm{~m}$ Very Large Telescope (VLT) of the European Southern Observatory at Paranal to obtain optical spectra of our candidate UCXBs. The observations were taken in the spring of 2003 and 2004.

\subsection{Results}

\section{U 1916-05}

In 2004 we obtained a spectrum of the known UCXB 4U 1916-05, which has a 50 min orbital period. In Fig. 3 we show the spectrum together with an LTE model of a disc of pure helium with (overabundant) nitrogen (black line) and a similar model with metals at solar abundance (grey line). We conclude that this is the first clear detection of a helium donor in an optical spectrum. However, the spectrum does not look at all like we had anticipated (i.e. strong helium emission lines, like the spectra of AM CVn stars ES Cet and GP Com). This means that really high $\mathrm{S} / \mathrm{N}$ spectra are needed to determine the chemical composition of the donors and that in our earlier results, the spectrum of $2 \mathrm{~S}$ 0918-549 


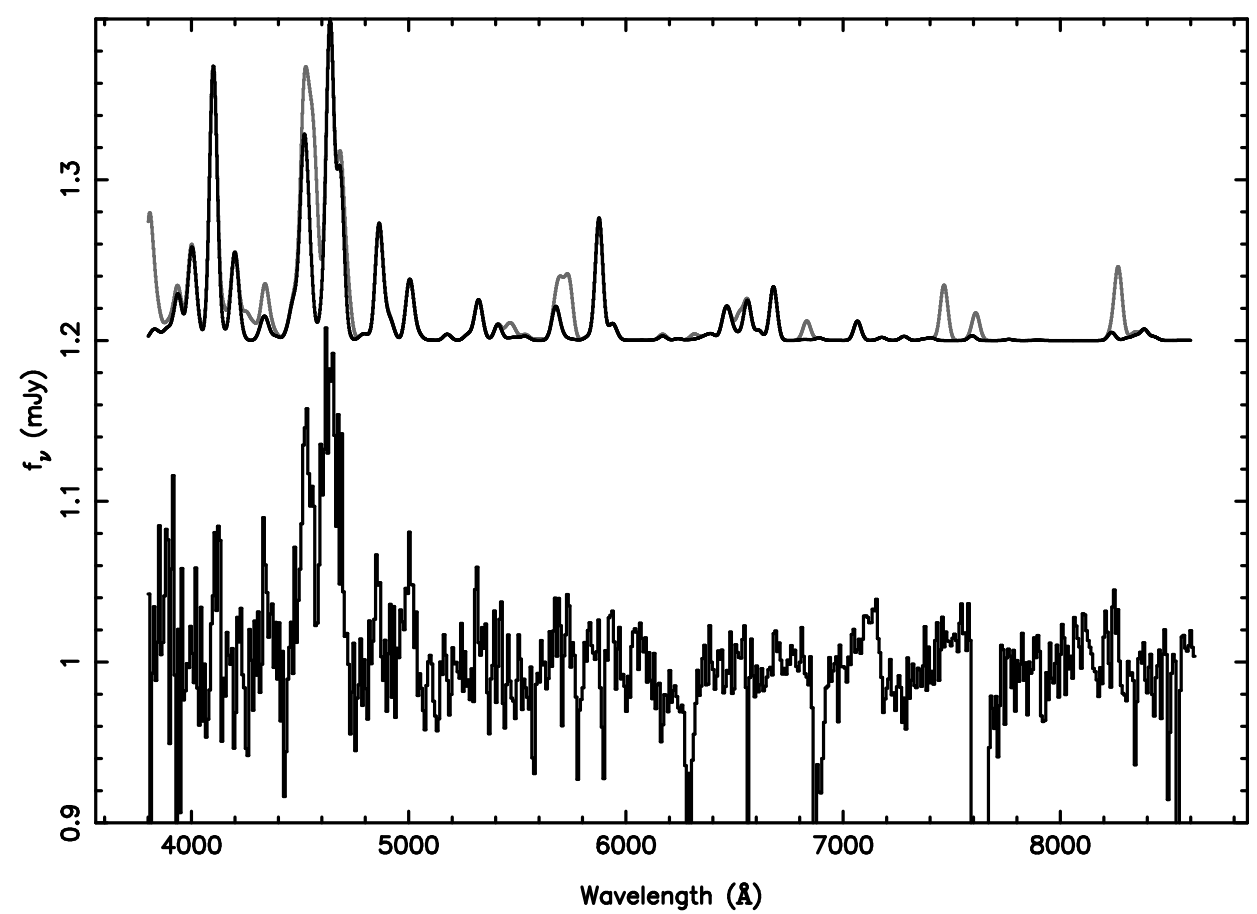

Fig. 3. VLT spectra of 4U 1916-05 (bottom). Above we show an LTE model with only helium and nitrogen (black line) and a similar model with all metals at solar abundance (grey line).

clearly is not good enough to distinguish between helium and $\mathrm{C} / \mathrm{O}$ and might be more likely a helium donor because of the properties of its X-ray bursts (in't Zand et al., 2005).

\section{XTE J0929-314}

XTE J0929-314 is one of the three transient UCXBs and was discovered in 2002 (Remillard et al., 2002). In outburst a spectrum was taken with the ESO $3.6 \mathrm{~m}$ telescope in which emission at $\mathrm{H} \alpha$ and around $4650 \AA$ was reported (Castro-Tirado et al., 2002). In particular the $\mathrm{H} \alpha$ emission is important, since this would clearly point to a donor with still some hydrogen left. However, the evidence for $\mathrm{H} \alpha$ emission is rather weak as is shown in Fig. 5, where we display the spectra. The formal significance of the $\mathrm{H} \alpha$ emission is $3 \sigma$, but one has to keep in

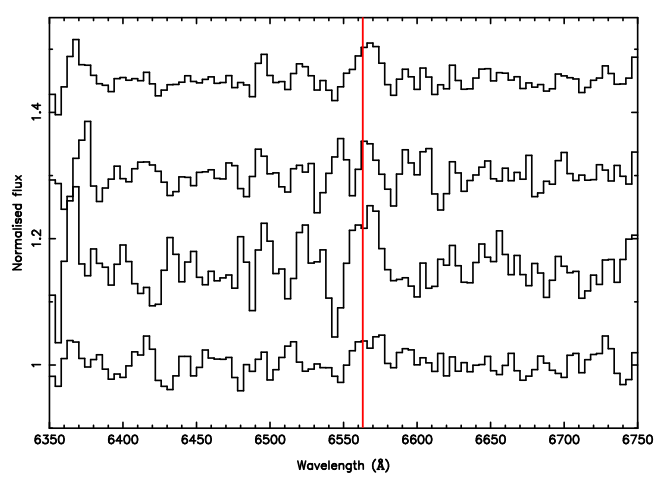

Fig. 5. Region around $\mathrm{H} \alpha$ of the outburst spectrum of XTE J0929-314 on the basis of which Castro-Tirado et al. (2002) claim $\mathrm{H} \alpha$ emission which would contradict the interpretation of the donors as white dwarfs. The 3 individual spectra are shown at the bottom, the average as the top spectrum.

mind that there are many emission lines in the night sky around $\mathrm{H} \alpha$ that could lead to systematic errors. The emission around $4650 \AA$ is clearly significant, as seen in Fig. 4, where we 


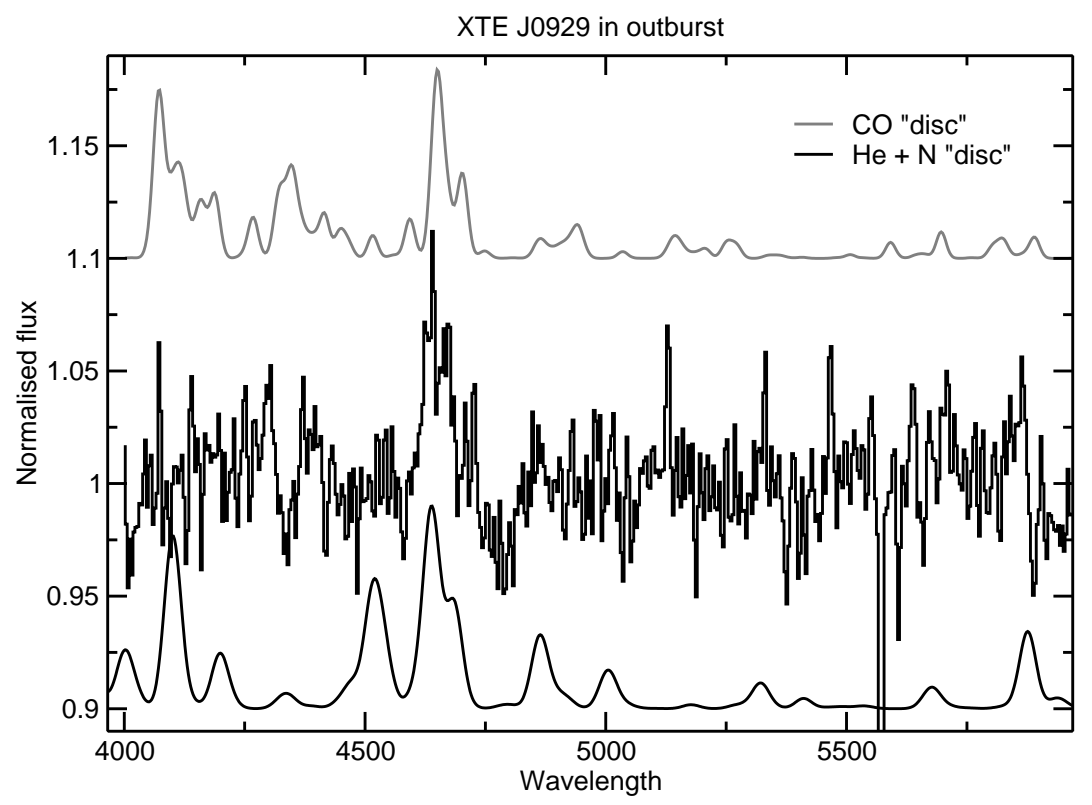

Fig. 4. Spectrum of XTE J0929-314 taken during outburst. Above and below an LTE model for a $\mathrm{CO}$ (top) and a $\mathrm{He} / \mathrm{N}$ (bottom) disc are shown.

also plot simple $\mathrm{C} / \mathrm{O}$ and He LTE model. The spectrum is not good enough to determine the composition, although the lack of emission around $4500 \AA$ suggest a $\mathrm{C} / \mathrm{O}$ donor.

\section{U 1626-67 and 4U 0614+09}

We also obtained a spectrum of $4 \mathrm{U}$ 1626-67. In Nelemans \& Jonker (2005) we already showed a comparison between the spectrum of $4 \mathrm{U}$ 1626-67 and our earlier 4U 0614+09 spectrum which looked very much like each other, in agreement with the interpretation of the donors of both these systems being $\mathrm{C} / \mathrm{O}$ rich. Indeed $4 \mathrm{U}$ 1626-67 follows the simple C/O LTE model that we used for $4 \mathrm{U} \quad 0614+09$ reasonably well even at bluer wavelengths than out $4 \mathrm{U} 0614+09$ spectrum reached. However, a second spectrum of $4 \mathrm{U}$ 0614+09 (obtained with the VLT by Klaus Werner, Werner et al. in prep.) with the same setup and wavelength coverage as our 4U 1626-67 spectrum clearly does not follow the simple LTE model! (see Fig. 6). It is clear that the last word on the interpretation of these spectra is not yet said.

\section{References}

Anderson, S. F., Haggard, D., Homer, L., et al. 2005, ApJ, submitted, astro-ph/0506730

Arons, J. \& King, I. R. 1993, ApJ, 413, L121

Castro-Tirado, A. J., Caccianiga, A., Gorosabel, J., et al. 2002, IAU Circ., 7895, 1

Cumming, A. \& Bildsten, L. 2001, ApJ, 559, L127

Dall'Osso, S., Luca Israel, G., \& Stella, L. 2005, A\&A, in press, astro-ph/0507474

Deloye, C. J. \& Bildsten, L. 2003, ApJ, 598, 1217

Deutsch, E. W., Margon, B., \& Anderson, S. F. 2000, ApJ, 530, L21

Dieball, A., Knigge, C., Zurek, D. R., 


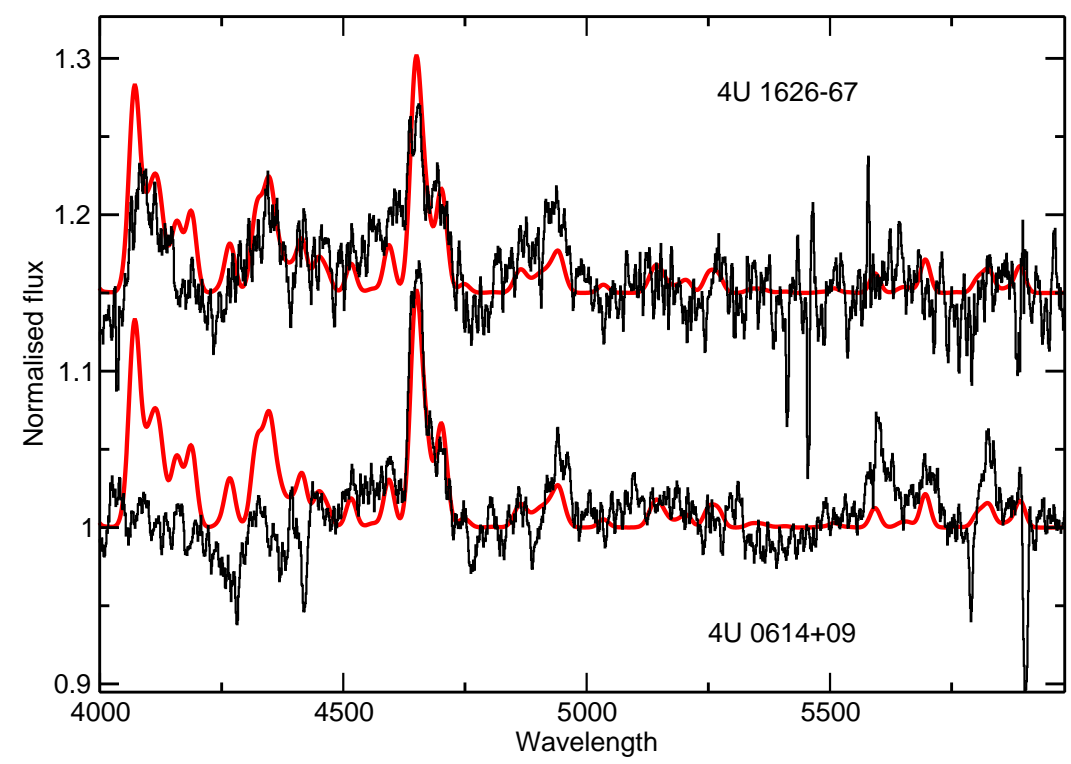

Fig. 6. Spectra of $4 \mathrm{U} 1626-67$ and $4 \mathrm{U} 0614+09$. The spectra are quite similar above $4500 \AA$, but below they are very different and interestingly the spectrum of $4 \mathrm{U} 1626-67$ follows the LTE model that we used for the line identification of 4U 0614+09 much better than $4 \mathrm{U} 0614+09$ itself!

et al. 2005, ApJ, in press, astro$\mathrm{ph} / 0510430$

Farinelli, R., Frontera, F., Masetti, N., et al. 2003, A\&A, 402, 1021

Hakala, P., Ramsay, G., Wu, K., et al. 2003, MNRAS, 343, 10

Heinke, C. O., Edmonds, P. D., \& Grindlay, J. E. 2001, ApJ, 562, 363

Homer, L., Charles, P. A., Naylor, T., et al. 1996, MNRAS, 282, L37

in't Zand, J. J. M., Cumming, A., van der Sluys, M. V., Verbunt, F., \& Pols, O. R. 2005, A\&A, 441, 675

Juett, A. M., Psaltis, D., \& Chakrabarty, D. 2001, ApJ, 560, L59

Kuulkers, E. 2005, The Astronomer's Telegram, 483

Kuulkers, E., den Hartog, P. R., in't Zand, J. J. M., et al. 2003, A\&A, 399, 663

Liu, Q. Z., van Paradijs, J., \& van den Heuvel, E. P. J. 2001, A\&A, 368, 1021
Markwardt, C. B., Swank, J. H., Strohmayer, T. E., Zand, J. J. M. i., \& Marshall, F. E. 2002, ApJ, 575, L21

Marsh, T. R. \& Nelemans, G. 2005, MNRAS, 363, 581

Nelemans, G. 2005, in ASP Conf. Ser. 330: The Astrophysics of Cataclysmic Variables and Related Objects, 27, astro-ph/0409676

Nelemans, G. \& Jonker, P. 2005, in Interacting binaries, ed. L. Antonelli, L. Burderi, F. D'Antona, T. Di Salvo, G. Israel, L. Piersanti, O. Straniero, \& A. Tornambè, AIP Conf. Proc. (New York: AIP), astro-ph/0412115

Nelemans, G., Jonker, P. G., Marsh, T. R., \& van der Klis, M. 2004, MNRAS, 348, L7

Nelemans, G., Portegies Zwart, S. F., Verbunt, F., \& Yungelson, L. R. 2001, A\&A, 368, 939

Nelson, L. A., Rappaport, S. A., \& 
Joss, P. C. 1986, ApJ, 304, 231

Norton, A. J., Haswell, C. A., \& Wynn, G. A. 2004, A\&A, 419, 1025

O'Brien, K., Jonker, P. G., Dhillon, V., et al. 2005, A\&A, in prep

Podsiadlowski, P., Rappaport, S., \& Pfahl, E. D. 2002, ApJ, 565, 1107

Poutanen, J. 2005, in Spectra \& Timing of Compact X-ray Binaries, astro-ph/0510038

Ramsay, G., Hakala, P., Wu, K., et al. 2005, MNRAS, 357, 49

Remillard, R. A., Swank, J., \& Strohmayer, T. 2002, IAU Circ., 7893, 1

Schulz, N. S., Chakrabarty, D., Marshall, H. L., et al. 2001, ApJ, 563, 941

Strohmayer, T. E. 2004, ApJ, 610, 416

—. 2005, ApJ, 627, 920

van Paradijs, J. \& McClintock, J. E. 1994, A\&A, 290, 133

Verbunt, F. 2005, in Interacting binaries, ed. L. Antonelli, L. Burderi, F. D'Antona, T. Di Salvo, G. Israel, L. Piersanti, O. Straniero, \& A. Tornambè, AIP Conf. Proc. (New York: AIP), astro$\mathrm{ph} / 0412524$

Verbunt, F. \& Lewin, W. H. G. 2005, in Compact Stellar X-ray Sources, ed. W. H. G. Lewin \& M. van der Klis (Cambridge: CUP), astroph/0404136

Wang, Z. \& Chakrabarty, D. 2004, ApJ, 616, L139

White, N. E. \& Angelini, L. 2001, ApJ, 561, L101

Wu, K., Cropper, M., Ramsay, G., \& Sekiguchi, K. 2002, MNRAS, 331, 221

Yungelson, L. R., Nelemans, G., \& van den Heuvel, E. P. J. 2002, A\&A, 388, 546 